\title{
Vigilância do desenvolvimento neuropsicomotor de crianças de um programa DST/AIDS
}

\author{
Neurodevelopmental surveillance of children participating in the STD/AIDS program
}

Aline Duprat Ramos ${ }^{1}$, Rosane Luzia de Souza Morais ${ }^{2}$

Estudo desenvolvido em municípios do Vale do Jequitinhonha, integrantes do Programa DST/AIDS da Secretaria Municipal de Saúde e Gerência Regional de Saúde de Diamantina (MG), Brasil.

${ }^{1}$ Fisioterapeuta do Hospital Pronto Socorro João XXIII (FHEMIG) - Belo Horizonte (MG), Brasil.

${ }^{2}$ Mestre; Professora Assistente do Departamento de Fisioterapia da Universidade Federal dos Vales do Jequitinhonha e Mucuri (UFVJM) Diamantina (MG), Brasil.

\section{ENDEREÇO PARA CORRESPONDÊNCIA}

Rosane Luzia de Souza Morais Departamento de Fisioterapia da UFVJM - Campus JK, 5000 - Alto da Jacuba - Rodovia MGT 367 - Km 583 - CEP: 39100-000 Diamantina - (MG), Brasil - E-mail: rosane.morais@ufvjm.com.br

\section{APRESENTAÇÃO}

mar. 2011

ACEITO PARA PUBLICAÇÃO set. 2011

\section{FONTE DE FINANCIAMENTO}

Pró-reitoria de Extensão e Cultura (PROEXC) da UFVJM, e apoios da Gerência Regional de Saúde e Programa DST/AIDS da Secretaria Municipal de Saúde de Diamantina (MG), Brasil

\section{CONFLITO DE INTERESSE}

nada a declarar

APRESENTAÇÃO EM EVENTO CIENTÍ́FICO

II Congresso Brasileiro de Fisioterapia na Saúde Coletiva, 2010 - Belo Horizonte (MG), Brasil

Parecer de aprovação no Comitê de Ética e Pesquisa da UFVJM (096/08)
RESUMO: A terapia anti-retroviral de alta potência (TARV) é uma forma eficaz de prevenção da transmissão do vírus HIV de mãe para filho. No entanto, os estudos ainda investigam os efeitos da exposição intraútero à TARV, dentre eles o atraso no desenvolvimento neuropsicomotor (DNPM). O presente estudo apresenta o relato de um projeto de extensão, cujos objetivos foram verificar o DNPM de crianças de um programa DST/AIDS, orientar as famílias considerando seu contexto socioeconômico e realizar encaminhamentos para serviços de saúde específicos. A vigilância do DNPM foi feita em três etapas: (1) avaliação em ambulatório; (2) avaliação e orientações em domicílio; (3) elaboração de relatórios aos gestores de saúde. Foram utilizados os testes DENVER II e o PEDI, além de um questionário socioeconômico. Participam do programa DST/AIDS 15 crianças, sendo 12 soro-revertidas, 1 soropositiva e 2 indefinidas. Doze crianças foram avaliadas, e os domínios mais comprometidos foram linguagem, pessoal-social e motor fino, respectivamente. Quanto ao nível econômico, 73,3\% pertenciam ao nível E, e 58,3\% das mães eram analfabetas ou cursaram apenas o primário. Crianças filhas de mães HIV positivo, além de fatores biológicos, geralmente estão expostas a fatores de risco ambientais que contribuem para alterações do DNPM. Desta forma, o acompanhamento por uma equipe de profissionais de saúde, em parceria com a família da criança, torna-se uma importante ferramenta para a identificação e intervenção precoce.

Descritores: desenvolvimento infantil; fatores de risco; ambiente; criança; infecções por HIV.

ABSTRACT: Highly active antiretroviral therapy (HAART) is an effective way of preventing mother-to-child transmission of HIV. However, further studies investigate the effects of short and long term exposure to HAART in-utero and its consequence on child neuropsychomotor development (NPMD). The paper presents a report and discussion of results of an extension project whose objectives were to verify the NPMD of children participating of the STD/AIDS program, to orientate families according to their socioeconomic context and make referrals to specific health services. The NPMD surveillance was divided into three parts: (1) ambulatory evaluation; (2) home evaluation and orientations; (3) reporting health managers. DENVER II and PEDI tests were used and also a socioeconomic questionnaire. Fifteen children were on the program of which 12 uninfected, $1 \mathrm{HIV}+$ and 2 indeterminate. Twelve children were evaluated and the most impaired domain were language, personal-social and fine motor, respectively. Regarding to socioeconomic status, 73,3\% were E level and $58,3 \%$ of mothers were analphabet or had primary school. Children born of infected mothers, besides the biological risks, usually are exposed to environment/social risks that can affect the NPMD. Thus, monitoring by a team of health professionals, in partnership with the child's family, becomes an important tool for identification and early intervention.

KeYwords: child development; risk factors; environment; child; HIV infections. 


\section{INTRODUCÃO}

Desde a instituição, em 1996, da terapia antirretroviral de alta potência (TARV) no Brasil durante o período pré, peri e pós-natal para prevenção da transmissão vertical do vírus, observou-se uma redução progressiva dos casos esperados de AIDS na população pediátrica. As crianças, apesar de nascidas de mães HIV positivo, não se contaminam com o vírus e, por isso, são chamadas sororrevertidas. Cerca de 6.000 novos casos de AIDS em crianças brasileira nascidas entre 1997 e 2001 foram evitados com a instituição da TARV' .

Apesar dos claros benefícios da TARV na prevenção da transmissão vertical do vírus, os efeitos cumulativos a curto e longo prazo da exposição intraútero no recém-nascido ainda encontra-se em investigação ${ }^{2-4}$. Dentre os possíveis efeitos citados, estão: disfunção mitocondrial persistente, hiperatividade, anemia reversível, baixo peso ao nascer, neutropenia, prematuridade e atraso no desenvolvimento neuropsicomotor (DNMP) ${ }^{2,5-8}$.

No entanto, quando se menciona atraso no DNMP em crianças expostas ao HIV, os fatores de risco biológicos não são a única causa ${ }^{9}$. É conhecido que crianças filhas de mães HIV positivo muitas vezes estão também expostas a riscos ambientais, como, por exemplo, condição de pobreza ${ }^{10,11}$ ou residência em orfanatos ${ }^{12,13}$.

O desenvolvimento infantil consiste em uma transformação complexa, contínua e dinâmica de domínios interdependentes. Os primeiros anos de vida são fundamentais para o desenvolvimento infantil em todos seus domínios: sensório motor, afetivo-social e cognitivo. Nessa fase do ciclo vital, o cérebro encontra-se em intensa organização ${ }^{14}$. Perturbações no ambiente físico, social, econômico e emocional podem interferir nesse processo, gerando consequências em longo prazo na capacidade funcional do cérebro da criança ${ }^{15}$. Sendo assim, a identificação precoce de fatores de risco para o DNPM, bem como uma adequada intervenção, são medidas de fundamental importância para a saúde da criança exposta ao vírus ${ }^{16}$.

Deste modo, o presente estudo apresenta o relato e discussão dos resultados de um projeto de extensão, cujos objetivos foram: (1) avaliar o DNPM de crianças do programa DST/AIDS da Secretaria Municipal de Saúde de Diamantina (SMSD), no Estado de Minas Gerais, em contexto de ambulatório e domicílio; (2) orientar as famílias, dentro da realidade socioeconômica em que vivem, quanto aos estímulos adequados para o DNPM das crianças; (3) informar os gestores de saúde local sobre a situação das crianças participantes do programa; (4) encaminhar, aquelas que necessitassem, a um serviço de saúde específico.

\section{MATERIAL E MÉTODOS}

Participantes

Participaram deste estudo 12 das 15 crianças cadastradas no programa DST/AIDS da SMSD, que foram expostas ao HIV durante a gestação e submetidas à TARV intraútero e/ou durante as primeiras semanas de vida. Todos os responsáveis assinaram o Termo de Consentimento Livre e Esclarecido, e o estudo foi aprovado pelo Comitê de Ética em Pesquisa da Universidade Federal dos Vales do Jequitinhonha e Mucuri (UFVJM), (parecer n $n^{\circ}$ 096/08).

\section{Instrumentos}

Foi elaborado um questionário para conhecer o perfil clínico e socioeconômico da criança. Para a classificação econômica, foi aplicado o questionário da Associação de Empresas de Pesquisa ${ }^{17}$.

Para a avaliação do DNPM e o desempenho funcional foram utilizados o DENVER $I I^{18}$ e o Inventário de Avaliação Pediátrica de Incapacidade $(P E D I)^{19}$, respectivamente.

O DENVER II é um instrumento padronizado, utilizado para triagem de crianças com risco de atraso no desenvolvimento global. É subdividido em quatro domínios: pessoal-social, motor fino adaptativo, linguagem e motor grosso. Cada item avaliado é classificado como cautela, quando a criança não executa ou recusa-se a realizar atividade prevista para 75 a 90\% das crianças de sua idade; atraso, quando a criança não executa ou recusa-se a realizar atividade prevista para mais de $90 \%$ dos que têm sua idade. O resultado final é considerado normal quando a criança apresenta no máximo
1 cautela e nenhum atraso; suspeito, quando apresenta 1 atraso e/ou 2 ou mais cautelas; e anormal, quando apresenta 2 ou mais atrasos ${ }^{18}$.

O PEDI é uma escala pediátrica norte-americana traduzida e adaptada para uso no Brasil. Trata-se de uma entrevista estruturada com os responsáveis sobre o desempenho funcional em atividades da rotina diária de crianças de seis meses a sete anos e meio de idade. É composta de três partes: habilidade da criança; influência do cuidador, características do ambiente. A somatória das pontuações obtidas em cada escala resulta em um escore total bruto que, por sua vez, são transformados em escores normativos. Assim, é possível verificar se o desempenho da criança está de acordo (normal) ou abaixo (atraso) daquele esperado para crianças brasileiras da mesma idade ${ }^{19}$. No presente estudo, foram utilizadas apenas as escalas de autocuidado e função social, da parte de habilidade funcional, para complementar a triagem do desenvolvimento das crianças $^{19}$.

\section{Procedimentos}

A equipe foi formada por acadêmicas e professora da área da Saúde da Criança do Departamento de Fisioterapia da UFVJM. Inicialmente, a equipe foi treinada para a aplicação dos testes padronizados.

A partir de então, o projeto foi dividido em duas etapas: I) avaliação em ambulatório; II) avaliação em domicílio, a fim de melhor compreender a realidade das famílias. Na consulta ambulatorial, foram aplicados os questionários clínico e socioeconômico e o teste DENVER II. O PEDI foi aplicado em domicílio.

Uma carta convite foi enviada às mães contendo uma data para a avaliação ambulatorial das crianças. Para a realização das visitas domiciliares, ofícios foram enviados aos gestores municipais de saúde, sendo marcada uma data para que a equipe do projeto pudesse ser recebida e acompanhada por um representante da saúde local. Ao final de cada visita domiciliar, orientações quanto ao estímulo do DNPM foram dadas aos cuidadores das crianças, dentro das necessidades delas e respeitando-se o contexto familiar. 
Encaminhamentos para outros serviços foram discutidos com os pais e representantes da saúde locais. Relatórios contendo informações sobre o ambiente físico e social em que vive a criança, e também sobre seu DNPM, foram elaborados e enviados aos gestores municipais de saúde.

\section{Análise de dados}

Foi realizada a análise descritiva dos dados, média, desvio padrão, porcentagem e frequência utilizando o software aplicativo Microsoft Excel, versão 2007, Ink.

\section{RESULTADOS}

\section{Caracterização dos participantes}

A Tabela 1 apresenta a caracterização clínica e socioeconômica de todas as crianças participantes do programa DST/ AIDS. Das 15 crianças cadastradas no programa, 12 eram sororrevertidas, 1 soropositiva e 2 com diagnóstico indefinido. Houve uma predominância do sexo feminino (80\%), e a média da faixa etária foi de 4,06 anos, variando de um ano e quatro meses de idade a seis anos e dez meses de idade. Nenhuma das crianças era órfã de mãe, e todas residiam em núcleo familiar.

Com relação ao nível socioeconômico das famílias das crianças envolvidas, $80 \%$ enquadravam-se na classe econômica de nível mais baixo - E - e $20 \%$ como nível D. Quanto ao grau de escolaridade das mães participantes do programa DST/AIDS, 58,3\% eram analfabetas ou apenas com o ensino primário. Todas as crianças acima de seis anos frequentavam escola.

\section{Desenvolvimento das crianças}

A Tabela 2 apresenta os resultados dos testes DENVER II e PEDI das 12 crianças avaliadas*. Cinquenta por cento apresentaram resultado final do teste DENVER II como suspeito ou anormal, $50 \%$ apresentaram cautelas ou atrasos no domínio da linguagem, sendo 33\% com cautelas ou atrasos no domínio da

Tabela 1. Caracterização das crianças participantes do projeto DST/AIDS da SMS de Diamantina (MG), Brasil

\begin{tabular}{lcccccc}
\hline Participante & $\begin{array}{c}\text { Sorologia } \\
\text { HIV }\end{array}$ & $\begin{array}{c}\text { Idade } \\
\text { (anos })\end{array}$ & $\begin{array}{c}\text { Gênero } \\
\text { Escolaridade } \\
\text { materna }\end{array}$ & $\begin{array}{c}\text { Frequência } \\
\text { escola/creche }\end{array}$ & NSE \\
\hline 1 & - & $4 a 3 \mathrm{~m}$ & $\mathrm{M}$ & $\mathrm{Fl}$ & $\mathrm{N}$ & $\mathrm{E}$ \\
3 & + & $3 \mathrm{a} 2 \mathrm{~m}$ & $\mathrm{~F}$ & $\mathrm{~A}$ & $\mathrm{~N}$ & $\mathrm{E}$ \\
4 & - & $2 \mathrm{a} 5 \mathrm{~m}$ & $\mathrm{~F}$ & $\mathrm{~A}$ & $\mathrm{~N}$ & $\mathrm{E}$ \\
5 & - & $6 \mathrm{a} 3 \mathrm{~m}$ & $\mathrm{M}$ & $\mathrm{A}$ & $\mathrm{S}$ & $\mathrm{E}$ \\
6 & - & $3 \mathrm{a} 8 \mathrm{~m}$ & $\mathrm{~F}$ & $\mathrm{~A}$ & $\mathrm{~N}$ & $\mathrm{E}$ \\
7 & - & $6 \mathrm{a} 1 \mathrm{~m}$ & $\mathrm{M}$ & $\mathrm{A}$ & $\mathrm{S}$ & $\mathrm{E}$ \\
8 & - & $6 \mathrm{a} 10 \mathrm{~m}$ & $\mathrm{~F}$ & $\mathrm{MI}$ & $\mathrm{S}$ & $\mathrm{E}$ \\
9 & - & $6 \mathrm{a} 5 \mathrm{~m}$ & $\mathrm{~F}$ & $\mathrm{~A}$ & $\mathrm{~S}$ & $\mathrm{E}$ \\
10 & - & $2 \mathrm{a} 10 \mathrm{~m}$ & $\mathrm{~F}$ & $\mathrm{PC}$ & $\mathrm{N}$ & $\mathrm{D}$ \\
11 & indefinido & $3 \mathrm{a} 5 \mathrm{~m}$ & $\mathrm{~F}$ & $\mathrm{PC}$ & $\mathrm{N}$ & $\mathrm{E}$ \\
12 & - & $2 \mathrm{a} 3 \mathrm{~m}$ & $\mathrm{~F}$ & $\mathrm{Fl}$ & $\mathrm{S}$ & $\mathrm{E}$ \\
13 & - & $4 \mathrm{a} 6 \mathrm{~m}$ & $\mathrm{~F}$ & $\mathrm{Fl}$ & $\mathrm{N}$ & $\mathrm{E}$ \\
14 & - & $2 \mathrm{a} 8 \mathrm{~m}$ & $\mathrm{~F}$ & $\mathrm{FI}$ & $\mathrm{N}$ & $\mathrm{E}$ \\
15 & indefinido & $1 \mathrm{a} 4 \mathrm{~m}$ & $\mathrm{~F}$ & $\mathrm{Fl}$ & $\mathrm{N}$ & $\mathrm{D}$ \\
\hline
\end{tabular}

NSE: nível socioeconômico; a: anos; m: meses; F: feminino; M: masculino; Fl: Fundamental Incompleto; A: Analfabeta; MI: Médio Incompleto; PC: Primário Completo; S: sim; N: não motricidade fina e $25 \%$ no domínio do pessoal-social. Apenas 8,3\% das crianças apresentaram cautela no domínio da motricidade grossa. Quanto ao PEDI, todas apresentaram desempenho adequado na escala pessoal-social, e apenas $18,1 \%$ das crianças apresentaram atraso na escala de autocuidado.

DISCUSSÃO

A progressão da imunodeficiência causada pelo HIV em crianças infectadas verticalmente é mais rápida quando comparada à de outras crianças e adultos HIV positivos. Desde a introdução da TARV, o controle da replicação viral em recém-nascidos infectados foi possível, resultando em uma diminuição das morbidades e aumento na expectativa de vida dessas crianças $^{20}$. No presente estudo, apenas uma criança do programa era soropositiva e fazia uso da TARV. O seu desempenho no teste de triagem DENVER II foi classificado como normal. Já no resultado do teste para $P E D I$, obteve resultado desfavorável referente ao autocuidado.

Mesmo não contaminadas pelo HIV, crianças nascidas de mães soropositivas compõem um grupo de risco para atraso no $\mathrm{DNPM}^{21}$. Uma das possíveis causas, embora controverso, é a exposição intraútero às drogas antirretrovirais ${ }^{2,4,21}$.

Hokjindee et al. ${ }^{22}$ verificaram em um Programa de Prevenção da Transmissão Vertical na Tailândia que, das 143 crianças participantes nascidas de mães HIV positivo e economicamente desfavorecidas, 15,4\% apresentaram DNPM suspeito ou anormal de acordo com resultados do teste DENVER II, e o domínio motor grosso foi aquele com mais casos de suspeita de atraso. No presente estudo, metade das crianças apresentou o DNPM suspeito ou anormal de acordo com o teste DENVER II, e, diferentemente dos resultados encontrados por Hokjindee et al. ${ }^{22}$, o domínio com maior casos de suspeita de atraso foi o da linguagem.

Estudos atuais têm apresentado evidências de que o risco para o crescimento e atraso no desenvolvimento global em crianças filhas de mães HIV positivo esteja mais relacionado aos fatores de riscos ambientais que essas crianças estão geralmente expostas ${ }^{8,12,13,21,22}$. 
Tabela 2. Resultados dos testes aplicados

\begin{tabular}{|c|c|c|c|c|c|c|c|}
\hline \multirow[b]{2}{*}{ Participante } & \multicolumn{4}{|c|}{ DENVER II } & \multicolumn{3}{|c|}{ PEDI } \\
\hline & $\begin{array}{l}\text { Pessoal- } \\
\text { social }\end{array}$ & $\begin{array}{l}\text { Motricidade } \\
\text { fina }\end{array}$ & $\begin{array}{c}\text { Motricidade } \\
\text { grossa }\end{array}$ & Linguagem & $\begin{array}{l}\text { Resultado } \\
\text { final }\end{array}$ & Autocuidado & $\begin{array}{c}\text { Pessoal } \\
\text { social }\end{array}$ \\
\hline 1 & $1 \mathrm{C}$ & $\mathrm{N}$ & $\mathrm{N}$ & $1 \mathrm{C}$ & $\mathrm{S}$ & AN & $\mathrm{N}$ \\
\hline 2 & $\mathrm{~N}$ & $\mathrm{~N}$ & $\mathrm{~N}$ & $1 \mathrm{C}$ & $\mathrm{N}$ & AN & $\mathrm{N}$ \\
\hline 3 & $2 \mathrm{C} 1 \mathrm{~A}$ & $1 \mathrm{C}$ & $\mathrm{N}$ & $2 \mathrm{CE} 4 \mathrm{~A}$ & AN & $\mathrm{N}$ & $\mathrm{N}$ \\
\hline 4 & $\mathrm{~N}$ & $\mathrm{~N}$ & $\mathrm{~N}$ & $\mathrm{~N}$ & $\mathrm{~N}$ & $\mathrm{~N}$ & $\mathrm{~N}$ \\
\hline 5 & $\mathrm{~N}$ & $\mathrm{~N}$ & $\mathrm{~N}$ & $\mathrm{~N}$ & $\mathrm{~N}$ & $\mathrm{~N}$ & $\mathrm{~N}$ \\
\hline 6 & $\mathrm{~N}$ & $\mathrm{~N}$ & $\mathrm{~N}$ & $\mathrm{~N}$ & $\mathrm{~N}$ & $\mathrm{~N}$ & $\mathrm{~N}$ \\
\hline 7 & $\mathrm{~N}$ & $\mathrm{~N}$ & $\mathrm{~N}$ & $\mathrm{~N}$ & $\mathrm{~N}$ & $\mathrm{~N}$ & $\mathrm{~N}$ \\
\hline $8^{*}$ & NR & NR & NR & NR & NR & NR & NR \\
\hline 9 & $\mathrm{~N}$ & $\mathrm{~N}$ & $\mathrm{~N}$ & $\mathrm{~N}$ & $\mathrm{~N}$ & $\mathrm{~N}$ & $\mathrm{~N}$ \\
\hline $10^{*}$ & NR & NR & NR & NR & NR & NR & NR \\
\hline 11 & $1 \mathrm{C}$ & $1 \mathrm{C}$ & $\mathrm{N}$ & $1 \mathrm{C}$ & AN & $\mathrm{N}$ & $\mathrm{N}$ \\
\hline 12 & $\mathrm{~N}$ & $1 \mathrm{~A}$ & $\mathrm{~N}$ & $1 \mathrm{~A}$ & S & $\mathrm{N}$ & $\mathrm{N}$ \\
\hline 13 & $\mathrm{~N}$ & $\mathrm{~N}$ & $\mathrm{~N}$ & $1 \mathrm{C}$ e $1 \mathrm{~A}$ & AN & $\mathrm{N}$ & $\mathrm{N}$ \\
\hline 14 & $\mathrm{~N}$ & $1 \mathrm{C} \mathrm{e} 1 \mathrm{~A}$ & $1 \mathrm{C}$ & $\mathrm{N}$ & $\mathrm{S}$ & $\mathrm{N}$ & $\mathrm{N}$ \\
\hline $15^{*}$ & NR & NR & NR & NR & NR & NR & NR \\
\hline
\end{tabular}

* Avaliação não foi realizada por não ter sido possível o contato prévio com a equipe local de saúde; C: cautela; N: normal; A: atraso; NR: não realizado; S: suspeito; AN: anormal

Williams et al. ${ }^{21}$ realizaram uma coorte com 1.840 crianças sororrevertidas, sendo que dessas 1.694 foram expostas à TARV intraútero. Os autores avaliaram e compararam o desempenho motor e cognitivo dessas crianças com crianças sororrevertidas não expostas à TARV intraútero aos 2 anos de idade. Concluíram que, ao ajustarem os achados em relação ao nível socioeconômico e dados demográficos, as crianças expostas à TARV intraútero não apresentaram desempenho inferior às crianças não expostas.

No presente estudo, a maioria das crianças pertencia a famílias economicamente desfavorecidas, e suas mães apresentavam baixo nível de escolaridade. Em situação de recursos financeiros escassos, há uma menor oferta de brinquedos, livros, investimentos em viagens e educação complementar, configurando assim um ambiente menos estimulante para o desenvolvimento ${ }^{23}$. Os efeitos da pobreza sobre a família não estão apenas relacionados à renda baixa, mas também a condições associadas ao nível socioeconômico baixo como baixa escolaridade dos pais, família numerosa, mães solteiras, pais adolescentes, entre outros $^{24,25}$.

Sendo assim, as crianças participantes deste estudo se enquadram no grupo de risco para atraso no DNPM por estarem expostas tanto a riscos biológicos quanto a riscos ambientais. Portanto, é importante o monitoramento do DNPM dessas crianças por profissionais da saúde em parceira com a família. Segundo Richter $^{26}$, a família é a unidade básica para a criança e tem o papel vital de assegurar-lhe a saúde e o bem-estar antes, durante e após uma intervenção realizada por uma equipe de saúde.

Neste estudo, a maioria das crianças recebeu visita domiciliar da equipe do projeto. As orientações passadas à família quanto aos adequados estímulos do DNPM da criança foram baseadas no resultado do desempenho do teste de cada criança, levando-se em consideração os anseios dela e de sua família, além do contexto socioeconômico em que viviam. Segundo o Institute For
Patient- and Family-Centered Care ${ }^{27}$, o profissional de saúde deve entender os valores, crenças e cultura da família, a fim de obter um melhor planejamento e qualidade no serviço, pois é ela que também fornece o apoio emocional e social ao desenvolvimento da criança, sendo assim parte integrante dos cuidados em saúde. Os encaminhamentos para outros profissionais foram realizados, quando necessário, e a partir de discussão com a família e agente de saúde local.

Os relatórios sobre os achados foram elaborados pela equipe do projeto e enviados aos gestores municipais de saúde. A documentação dos achados provê aos gestores informações que permitem o aperfeiçoamento e/ou redirecionamento de suas ações em saúde, além de endossar propostas que visem à intervenção para a qualificação da atenção à saúde da criança. Tais aspectos contemplam as diretrizes operacionais do Pacto pela Vida e do Pacto de Gestão, propostos pelo Ministério da Saúde do Brasil ${ }^{28}$.

* Três das 15 crianças não foram avaliadas por não ter sido possível o contato prévio com a equipe local de saúde. 


\section{LIMITAÇÕES DO ESTUDO}

Os resultados do presente estudo são específicos para as crianças participantes do programa de DST/AIDS investigado. No entanto, esses achados, respaldados na literatura recente, são importantes para fomentar a discussão sobre o risco para atraso no DNPM em crianças sororrevertidas. Nesse caso, há necessidade de vigilância e intervenção fisioterapêutica, bem como de outros profissionais da saúde.

É importante que em estudos futuros, além do DNPM, essas crianças sejam acompanhadas quanto à exposição a outros riscos relatados na literatura, tais como morbidades e o estado nutricional deficiente ${ }^{8,22}$.

\section{CONCLUSÃO}

Crianças filhas de mães HIV positivo, sororrevertidas, além do fator biológico, geralmente estão expostas a fatores ambientais de risco para o DNPM. Dessa forma, o acompanhamento por uma equipe de profissionais de saúde, em parceria com a família da criança, tornase uma importante ferramenta para a identificação e intervenção precoce nas alterações no DNPM.

\section{AGRADECIMENTOS}

Agradecemos a participação das acadêmicas Gabriela Silveira Neves, Fernanda Ribas Bernardes e Raquel Mara Vieira Nogueira, o apoio fornecido pelo Programa DST/AIDS da Secretaria Municipal de Diamantina (MG), Gerência Regional de Saúde de Diamantina (MG) e os Gestores de saúde das cidades visitadas pela nossa equipe.

\section{REFERÊNCIAS}

1. Brito AM, Sousa JL, Luna CF, Dourado I. Tendência da transmissão vertical de Aids após terapia antirretroviral no Brasil. Rev Saude Publica. 2006;40(Suppl):9-17.

2. Poirier MC, Divi RL, Al-Harthi L, Olivero OA, Nguyen $\mathrm{V}$, Walker B, et al. Long-term mitochondrial toxicity in HIVuninfected infants born to HIV-infected mothers. J Acquir Immune Defic Syndr. 2003;33(2):175-83.

3. El Beitune P, Duarte G, Quintana SM, Figuieró-Filho EA, Marcolin AC, Abduch R. Antiretroviral therapy during pregnancy and early neonatal life: consequences for HIV-exposed, uninfected children. Braz J Infect Dis. 2004;8(2):140-50.

4. Briand N, Mandelbrot L, Le Chenadec J, Tubiana R, Teglas JP, Faye A, et al. No relation between in-utero exposure to HAART and intrauterine growth retardation. AIDS. 2009;23(10):1235-43.

5. Alimenti A, Forbes JC, Oberlander TF, Money DM, Grunau RE, Papsdorf MP, et al. A prospective controlled study of neurodevelopment in HIV-uninfected children exposed to combination antiretroviral drugs in pregnancy. Pediatrics. 2006;118(4):1139-45.

6. Feiterna-Sperling C, Weizsaecker K, Bührer C, Casteleyn S, Loui A, Schmitz T, et al. Hematologic effects of maternal antiretroviral therapy and transmission prophylaxis in HIV-1-exposed uninfected newborn infants. J Acquir Immune Defic Syndr. 2007;45(1):43-51.

7. Townsend CL, Cortina-Borja M, Peckham CS, Tookey PA. Antiretroviral therapy and premature delivery in diagnosed HIV-infected women in the United Kingdom and Ireland. AIDS. 2007;21(8):1019-26.

8. Powis KM, Smeaton L, Ogwu A, Lockman S, DrydenPeterson S, van Widenfelt $E$, et al. Effects of in utero antiretroviral exposure on longitudinal growth of HIV-exposed uninfected infants in Botswana. J Acquir Immune Defic Syndr. 2011;56(2):131-8.

9. Resegue R, Puccini RF, Silva EMK. Fatores de risco associados a alterações no desenvolvimento da criança. Pediatria. 2007;29(2):117-28.

10. Walker SP, Wachs TD, Gardner JM, Lozoff B, Wasserman GA, Pollitt E, et al. Child development: risk factors for adverse outcomes in developing countries. Lancet. 2007;369(9556):145-57.

11. Boutayeb A. The impact of HIV/AIDS on human development in African countries. BMC Public Health. 2009;9(Suppl 1):S3.

12. Dobrova-Krol NA, van IJzendoorn MH, BakermansKranenburg MJ, Juffer F. Effects of perinatal HIV infection and early institutional rearing on physical and cognitive development of children in Ukraine. Child Dev. 2010;81(1):237-51

13. Jelsma J, Davids N, Ferguson G. The motor development of orphaned children with and without HIV: Pilot exploration of foster care and residential placement. BMC Pediatr. 2011;11:11.

14. Thompson RA, Nelson CA. Developmental science and the media: Early brain development. Am Psychol. 2001;56(1):5-15.

15. Hackman DA, Farah MJ. Socioeconomic status and the developing brain. Trends Cogn Sci. 2009;13(2):65-73.

16. Willrich A, Azevedo CCF, Fernandes JO. Desenvolvimento motor na infância: influência dos fatores de risco e programas de intervenção. Rev Neurocienc. 2009;17(1):51-56.

17. Associação Brasileira de Empresas de Pesquisa [Internet]. Critério de Classificação Econômica Brasil 2009 [citado 


\section{Referências (cont.)}

2011 mar 28]. Disponível em: http://www.abep.org/ novo/Content.aspx?SectionID=84

18. Frankenburg WK, Dodds J, Archer P, Bresnick B, Maschka P, Edelman N, et al. Denver II: technical manual. 2nd ed. Denver: Denver Developmental Materials; 1992.

19. Mancini MC. Inventário de avaliação pediátrica de disfunção: versão brasileira. Belo Horizonte: Laboratórios de Atividade e Desenvolvimento Infantil. Departamento de Terapia Ocupacional. Universidade Federal de Minas Gerais; 2000.

20. de Martino M, Tovo PA, Balducci M, Galli L, Gabiano C, Rezza G, et al. Reduction in mortality with availability of antiretroviral therapy for children with perinatal HIV-1 infection. Italian Register for HIV Infection in Children and the Italian National AIDS Registry. JAMA. 2000;284(2):190-7.

21. Williams PL, Marino M, Malee K, Brogly S, Hughes MD, Mofenson LM, et al. Neurodevelopment and in utero antiretroviral exposure of HIV-exposed uninfected infants. Pediatrics. 2010;125(2):e250-60.

22. Hokjindee U, Chongsuvivatwong V, Lim A, Pruphetkaew N. Denver Developmental Screening Test (DDST) survey and degree of malnutrition among children born to HIV infected mothers under the Prevention of Mother toChild-Transmission (PMTCT) Program. J Med Assoc Thai. 2010;93(12):1458-62.
23. Engle PL, Black MM, Behrman JR, Mello MC, Gertler PJ, Martorell R, Young ME, Strategies to avoid the loss of developmental potential in more than 200 million children in the developing world. Lancet. 2007;369(9557):229-42.

24. Andrade SA, Santos DN, Bastos AC, Pedromônico MRM, de Almeida-Filho N, Barreto ML. Ambiente familiar e desenvolvimento cognitivo infantil: uma abordagem epidemiológica. Rev Saude Publica. 2005;39(4):606-11.

25. Assis SG, Avanci JQ, Vasconcellos R, de Oliveira CVC. Desigualdades socioeconômicas e saúde mental infantil. Rev Saude Publica. 2009;43(Supl. 1):92-100.

26. Richter L. An introduction to family-centred services for children affected by HIV and AIDS. J Int AIDS Soc. 2010;13(Suppl 2):S1.

27. Institute For Patient- and Family-Centered Care [Internet]. Partnering with patients and families to design a patient-and family-centered health care system: recommendations and promising practices. 2008 [citado 2011 mar 28]. Disponível em: http://www.ipfcc.org/faq.html.

28. Ministério da Saúde. Diretrizes operacionais para os Pactos pela Vida, em Defesa do SUS e de Gestão. Brasília; 2005 (Série A. Normas e Manuais Técnicos). 72 p. 